International Journal on Cybernetics \& Informatics (IJCI) Vol. 5, No. 1, February 2016

\title{
PDE BASED Features For TeXTURE ANALYsis USING WAVELET TRANSFORM
}

\author{
P. S. Hiremath ${ }^{1}$ and Rohini A. Bhusnurmath ${ }^{2}$ \\ ${ }^{1}$ Department of Computer Science (MCA), KLE Technological University, \\ BVBCET Campus, Hubli-580031, Karnataka, India. \\ ${ }^{2}$ Department of P.G. Studies and Research in Computer Science, \\ Gulbarga University, Gulbarga-585106, Karnataka, India.
}

\begin{abstract}
In the present paper, a novel method of partial differential equation (PDE) based features for texture analysis using wavelet transform is proposed. The aim of the proposed method is to investigate texture descriptors that perform better with low computational cost. Wavelet transform is applied to obtain directional information from the image. Anisotropic diffusion is used to find texture approximation from directional information. Further, texture approximation is used to compute various statistical features. LDA is employed to enhance the class separability. The $k-N N$ classifier with tenfold experimentation is used for classification. The proposed method is evaluated on Brodatz dataset. The experimental results demonstrate the effectiveness of the method as compared to the other methods in the literature.
\end{abstract}

\section{KEYWORDS}

Anisotropic Diffusion, Wavelet Transform, Texture Approximation, Partial Differential Equation (PDE).

\section{INTRODUCTION}

Texture is a recurring pattern in image intensity. Texture can be defined as a similarity grouping in an image [1]. Texture analysis represents mathematical approach that captures gray level variations in an image. Texture perception is a function of radiometric and spatial scales. It is used in computer vision and image analysis. An image is the matrix of pixels and a texture in an image can be considered as descriptors of interrelated pixels. Tuceryan and Jain [2] categorized texture analysis approaches into statistical, signal processing, geometrical, and model based methods. The study of human vision system reveals that spatial or frequency representation preserving local and global information is suitable for quasi periodic signal. Haralick et al. [3] proposed gray level co-occurrence matrix (GLCM) for texture feature extraction. This approach explored spatial gray level dependence of texture. Tamura et al. [4] proposed a computation of six visual properties like contrast, coarseness, likeliness, regularity, directionality and roughness. In [5] wavelet transform is used to obtain features for texture classification. Smith and Chang [6, 7] used the statistical features such as mean and variance from wavelet sub-bands as texture measures. The local directional binary patterns (LDBP) and non-subsampled contourlet transform (NSCT) based texture classification using k-NN classifier is introduced in [8]. The effective LDBP's are investigated which characterize local image texture [9]. In [10], support vector machine is used for rotation and shift invariant texture classification. Thyagarajan et al. [11] proposed wavelet transform with co-occurrence matrix for texture analysis. The commonly used texture descriptors that have been used successfully to real-world textures are the Laws' texture energy measures [12] and Fourier power spectrum [13]. The fractal concept developed by Mandelbrot [14] provides an excellent representation of the roughness of natural textural surfaces. The partial differential equation (PDE) based anisotropic diffusion filter is used for image processing. The PDE techniques are widely used in image and signal processing [15]. Most DOI: $10.5121 /$ ijci.2016.5114 
International Journal on Cybernetics \& Informatics (IJCI) Vol. 5, No. 1, February 2016

methods based on PDE are used to smooth the image while preserving the edges [16]. An effective method for texture classification using local directional binary pattern co-occurrence matrix and anisotropic diffusion is presented in [17]. Anisotropic diffusion for color texture image classification is explored on RGB color space [18]. Extraction of LDBP features using diffusion approach on different datasets is presented in [19]. Randen and Husoy [20] concluded in a survey of filtering methods that future direction of research is extraction and classification of powerful texture features with low computational cost. This observation has inspired to develop proposed method.

The combination of transform based method and statistical method for feature extraction is proposed in the paper. The objective of this paper is to obtain better classification accuracy with reduced computational cost. Wavelet transform is used to obtain directional information. Anisotropic diffusion is applied on directional information to find texture approximation. Further, various statistical features are computed from texture approximation. Linear discriminant analysis (LDA) enhances the class separability. The k-NN classifier is used for classification. The method is evaluated on sixteen texture classes from Brodatz image dataset. The average classification accuracies obtained by using various statistical feature sets are compared with other methods in the literature.

\section{Proposed Method}

The proposed method comprises the following steps:

i. Apply Haar wavelet transform on input image to yield H, V and D subbands.

ii. Apply anisotropic diffusion on $\mathrm{H}, \mathrm{V}$ and $\mathrm{D}$ components up to $\mathrm{n}$ diffusion steps and obtain texture approximation.

iii. Extract statistical features from texture approximation image and use LDA on feature sets for optimization

iv. Use k-NN classifier to classify the feature set of textural image.

These methods are described briefly as given below.

\subsection{Wavelet Transform}

During the past decades wavelet analysis has become a powerful tool for multi-resolution analysis. Intuitively, multiscale wavelet analysis is an ideal approach to analyze texture because it is well recognized that scale is one of the most important aspects of texture information. The wavelet based methods are computationally effective over other methods for the texture classification. The different wavelet transform functions filter out different range of frequencies (i.e., subbands). Thus, wavelet is a powerful tool, which decomposes the image into low frequency and high frequency subband images. The implementation and theoretical aspects of wavelet based algorithms are discussed in [21, 22, 23]. Time dependent signal analysis at different resolution is achieved by wavelet transform. The wavelet transform has several advantages making it attractive for texture analysis. That include -textures are represented at the suitable scale in varying the spatial resolution. Wavelet functions are available in wide range of choices, so that wavelet best suited for texture analysis can be chosen in a specific application. In an image, the wavelet decomposition is obtained with separable filtering along the columns and the rows and of an image [23]. The decomposition of a signal into a set of detail coefficients $(\mathrm{H}$, $\mathrm{V}, \mathrm{D})$ and an approximation coefficient (A) is obtained in discrete wavelet transform. The D subband represents diagonal details (high frequencies in both directions - the corners), $\mathrm{H}$ gives horizontal high frequencies, $\mathrm{V}$ gives vertical high frequencies, and the image A corresponds to the lowest frequencies. The Figure 1 represents the level 1 (1-scale) image decomposition. The original Brodatz texture image D51 is shown in the Figure 1(b) and its 1-level Haar wavelet transform is shown in the Figure 1(c). 


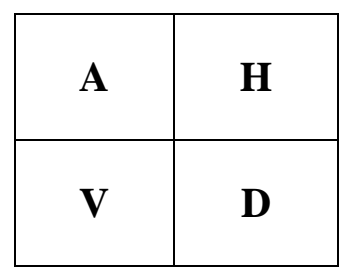

(a)

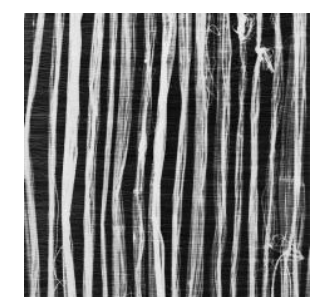

(b)

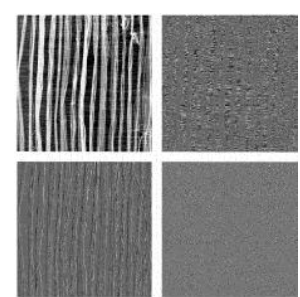

(c)

Figure 1. Wavelet transform (a) Subbands of level 1 decomposition (b) Brodatz texture image D51 (c) 1level Haar wavelet transform of the image in (b).

\subsection{Anisotropic Diffusion}

Anisotropic diffusion filter proposed by Perona - Malik [15] is used to smooth an image without altering important parts of the image such as edges. The edge detection is done using scale space approach. The family of parameterized images is produced in combination of original image and image dependent filter. This makes anisotropic diffusion a space variant and nonlinear transformation of the image. The relation between filtered image $F(x, y, t)$ and the original image $\mathrm{F}_{0}(\mathrm{x}, \mathrm{y})$ is represented by $\mathrm{F}(\mathrm{x}, \mathrm{y}, \mathrm{t})=\mathrm{F}_{0}(\mathrm{x}, \mathrm{y}) * \mathrm{GK}(\mathrm{x}, \mathrm{y}, \mathrm{t})$, where $\mathrm{GK}(\mathrm{x}, \mathrm{y}, \mathrm{t})$ is a Gaussian kernel having variable scale parameter $t$. The filtering is the result of iterative heat diffusion. The initial condition is given by the equation $\mathrm{F}(\mathrm{x}, \mathrm{y}, 0)=\mathrm{F}(\mathrm{x}, \mathrm{y})$, where $\mathrm{t}$ represents time. The diffusion process smoothes the image and is stopped at the edges and boundaries. The method is described in brief in Appendix.

\subsection{Statistical Features}

Statistical methods are used to compute local features by considering gray values in an image [24]. Statistical methods are classified into first order (one pixel), second order (two pixels) and higher order (more than two pixels) statistics [24]. The first-order statistics captures properties (e.g. average and variance) considering individual pixel values, whereas second and higher order statistics represent the spatial dependency between two or more pixel values relative to each other. Various feature vectors considered for experimentation are described below.

\subsubsection{First order statistics for texture analysis (F1)}

First order texture features are calculated from the gray values of original image. These statistics do not consider relationships between neighboring pixels. Five features, namely, mean, median, standard deviation, skewness and kurtosis are considered for experimentation. Texture analysis based on these statistical features lacks the information about the relationship between neighboring pixels.

\subsubsection{Haralick features (F2)}

Haralick et al. [3] suggested second order statistics of textures that considers relationship between pairs of pixels. They used gray level co-occurrence matrices (GLCM) which have become well known and widely used textural features. GLCM shows a function of joint probability distributions of pairs of gray level pixels. The most commonly used seven textural features, namely, contrast, energy, entropy, homogeneity, maximum probability, cluster shade and cluster prominence over angles $0,45,90$ and 135 degrees ( 7 features $\times 4$ angles $=28$ number) are considered for the experimentation. 


\subsubsection{Gray level difference statistics (F3)}

This method is described in [25]. Five features, namely, homogeneity, contrast, energy, entropy and mean are calculated from a single grey level difference probability distribution vector. These features are obtained from sum of four vectors for 0, 45, 90 and 135 degrees. Methods based on second-order statistics have higher discrimination capability than the structural and power spectrum (transform based) methods [25].

\subsubsection{Neighborhood gray tone difference matrix (F4)}

Image texture can be represented as a function of the structural and tonal relationships between the pixels. Tone is based mainly on pixel intensity (gray values), while the structure is the spatial (location) relationship between pixels [26]. A Grey-Tone Difference Matrix (GTDM) was proposed in [27] to correlate texture measures with human perception of textures. A GTD matrix is a vector containing $\mathrm{G}$ elements. Its elements are computed as the difference between gray value of pixel and average of gray values over a square by sliding the window centered at the pixel. Features, namely, coarseness, contrast, busyness, complexity, and texture strength are considered.

\subsubsection{Statistical feature matrix (F5)}

This method of feature extraction was introduced in [28]. Four features, namely, coarseness, contrast, period and roughness are considered.

\subsubsection{Law's texture energy measures (F6)}

This method is described in [29, 30]. Six texture energy measures are given by standard deviation of convolved image over entire region of interest. The measures are derived from three simple vectors. $\mathrm{L} 3=(1,2,3)$ which represents averaging; $\mathrm{E} 3=(-1,0,1)$ calculating first difference (edges); and $\mathrm{S} 3=(-1,2,-1)$ corresponding to the second difference (spots). Mutually multiplying these vectors by considering the first term as a column vector and the second term as row vector, results in 3 X 3 matrix known as Law's masks. The Law's mask is convolved over texture image and six energy statistics are calculated, which are used for texture description.

\subsubsection{Fractal dimension texture analysis (F7)}

Texture analysis based on fractal was introduced in [14]. The geometric complexity of spatial pattern of textures is represented using fractals [14]. The word 'fractal' refers to complex patterns that re-occur at various scales and are independent of scales. Fractal metrics provide features patterns of self similarity at different scales. The fractal dimension gives a global descriptor of complexity or irregularity of a geometric object. It has been found that fractal dimension encode textures in nature, which represents the irregularity of textures. The fractal dimension is used to calculate roughness of a surface. The larger the fractal dimension, the rougher is the texture.

\subsubsection{Fourier power spectrum (F8)}

Texture descriptors are scale dependant. A texture is described in multiple resolutions to decrease the scale sensitivity. An appropriate scale to achieve the maximum texture discrimination may be chosen. For calculating multiscale feature, time-frequency method known as Fourier spectral method [31] is adopted. It is an image in a space whose co-ordinate system has an interpretation that is closely related to the characteristics of a texture (such as size or frequency). Two features, namely, radial sum and angular sum features are computed. 


\subsubsection{Shape (F9)}

Five shape features: size $(\mathrm{x}, \mathrm{y})$, area, perimeter and perimeter ${ }^{\wedge} 2$ /area are considered for experimentation. Texture size measure may be based on co-occurrences of edge pairs with opposite edge directions at constant distance in a direction perpendicular to the edge directions. These statistics are derived from the second order statistics.

\section{Feature Extraction And TeXture Classification}

Feature extraction is the important stage of texture analysis. Features obtained from this stage are used for texture classification.

\subsection{Feature extraction}

The steps of the proposed method are given in the Algorithm 1.

\begin{tabular}{|c|c|}
\hline \multicolumn{2}{|c|}{ Algorithm 1: Training Algorithm } \\
\hline Step 1: & Read the training image block I. \\
\hline Step 2 & $\begin{array}{l}\text { Decompose the image, using Haar wavelet transform, into horizontal (H), } \\
\text { vertical (V) and diagonal (D) components. }\end{array}$ \\
\hline Step 3: & Subject the H, V and D components to anisotropic diffusion. \\
\hline Step 4 & $\begin{array}{l}\text { Obtain texture approximation } \mathrm{I}_{\mathrm{Htxr},} \mathrm{I}_{\mathrm{Vtxr},} \mathrm{I}_{\mathrm{Dtxr}} \text { for } \mathrm{H}, \mathrm{V} \text { and } \mathrm{D} \text { components, } \\
\text { respectively. }\end{array}$ \\
\hline Step 5 : & $\begin{array}{l}\text { Compute statistical features (as in section 2.3) for images } I_{H t x r} I_{V t x r}, I_{D t x r} \text { obtained } \\
\text { in Step } 4 \text {, which form feature vector } F \text {. The } F \text { is stored in the feature database, } \\
\text { with class label. }\end{array}$ \\
\hline Step 6 : & $\begin{array}{l}\text { The Steps } 1-5 \text { are repeated for all the training blocks of all the texture class } \\
\text { images and the training feature set (TF) is obtained. }\end{array}$ \\
\hline Step 7 : & $\begin{array}{l}\text { LDA is applied on training feature set (TF) of Step } 6 \text {. The discriminant feature } \\
\text { set (TFLDA) is obtained, which is then used for texture classification. }\end{array}$ \\
\hline Step 8 & Stop. \\
\hline
\end{tabular}

The Algorithm 1 is executed up to different numbers of diffusion steps $(t)$ and extracted various features as discussed in section 2.3.

\subsection{Texture classification}

The texture classification is performed using k-NN classifier with ten-fold experimentation, based on Euclidean distance [32]. The steps of testing algorithm are given in the Algorithm 2.

Algorithm 2 : Testing Algorithm (Classification of test images)

Step 1: $\quad$ Read the test image block $\mathrm{I}_{\text {test. }}$

Step 2: Decompose the image $\mathrm{I}_{\text {test }}$, using Haar wavelet transform, into horizontal $(\mathrm{H})$, vertical (V) and diagonal (D) components.

Step 3: $\quad$ Subject the H, V and D components to anisotropic diffusion.

Step 4: Obtain texture approximation $\mathrm{I}_{\text {testHtxr, }} \mathrm{I}_{\text {testVtxr, }} \mathrm{I}_{\text {testDtxr }}$ for $\mathrm{H}, \mathrm{V}$ and D components, respectively.

Step 5: Compute statistical features (as in section 2.3) for images $I_{\text {testHtxr, }} I_{\text {testVtxr, }} I_{\text {testDtxr }}$ obtained in the Step 4, to form feature vector $\mathrm{F}_{\text {test }}$.

Step 6: Project $\mathrm{F}_{\text {test }}$ on LDA components stored in TFLDA and obtain the weights which constitute test image feature vector $\mathrm{F}_{\text {testLDA. }}$ 
International Journal on Cybernetics \& Informatics (IJCI) Vol. 5, No. 1, February 2016

Step 7: (Classification) The $\mathrm{k}-\mathrm{NN}$ classifier $(\mathrm{k}=3)$ based on Euclidean distance is employed to classify the test image $\mathrm{I}_{\text {test }}$ using $\mathrm{F}_{\text {testLDA }}$ vectors.

Step 8: Stop.

\section{EXPERIMENTAL RESUlts AND DisCuSSION}

\subsection{Dataset}

The sixteen texture images from Brodatz album [33] are considered for experimentation and these are shown in the Figure 2. Each Brodatz texture sample represents one class. Each texture image is of 256x256 pixels with 256 gray levels. The experimental dataset includes regular textures, namely, D4, D3, D36, D75, D16, D21, D68, D24, nonregular textures D82, D11, D29, D104, D71 and highly regular textures D6, D52, D51. Each texture image is subdivided into 16 non overlapping blocks of $64 \times 64$ pixels. Thus, totally 256 blocks are considered. The texture images in the dataset have different gray scale properties. The performance of unbiased texture classification is estimated by performing ten-fold experiments. The average of ten experimental results is reported in the paper. The images are randomly separated into distinct training and testing sets. The $50 \%$ of the sub images are taken as training set and remaining 50\% sub images are used for testing.
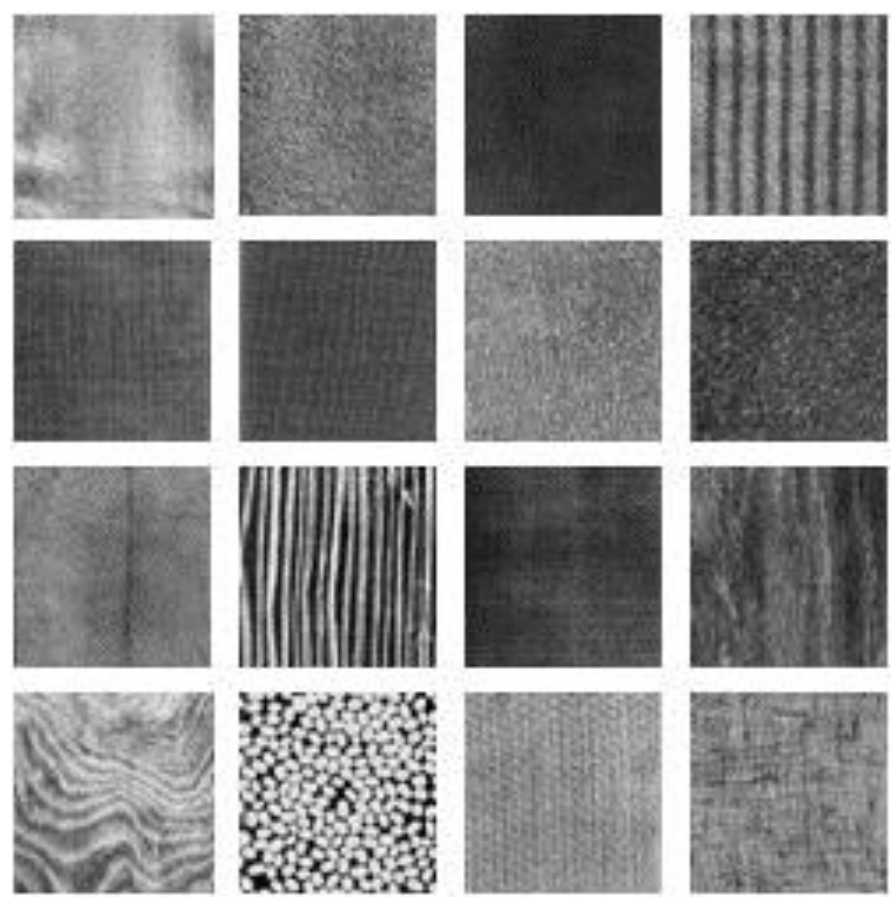

Figure 2. Texture images from Brodatz album from left to right and top to bottom: D3, D4, D6, D11, D16, D21, D24, D29, D36, D51, D52, D68, D71, D75, D82, D104.

\subsection{Experimental results}

The experimentation of the proposed method is carried out on Intel ${ }^{\circledR}$ Core $^{\mathrm{TM}}$ i3-2330M @ $2.20 \mathrm{GHz}$ with $4 \mathrm{~GB}$ RAM using MATLAB 7.9 software. The Haar wavelet transform is employed to decompose the image, resulting in average (A), horizontal $(\mathrm{H})$, vertical $(\mathrm{V})$ and diagonal (D) components. The $\mathrm{H}, \mathrm{V}$ and $\mathrm{D}$ components of the image are then subjected to anisotropic diffusion to find texture approximation. Further, different statistical features (discussed in section 2.3) are computed from the texture approximations. The LDA is used to 
International Journal on Cybernetics \& Informatics (IJCI) Vol. 5, No. 1, February 2016

enhance the class separability. The k-NN classifier is used for classification. The values for optimization of parameters of anisotropic diffusion are: lambda $=0.25$ and conduction coefficient $=60$. The experimentation for each feature set is executed up to 10 diffusion steps. The average classification accuracy is computed for the sixteen class problem, where 16 texture classes are considered for the experimentation from Brodatz texture dataset [33].

The Table 1 shows the comparison of average classification accuracy, average training time and average testing time for various feature sets using the optimal number of diffusion steps. Totally, thirty feature sets (including combinations of feature sets in section 2.3) are taken for experimentation. The optimal number of diffusion step is the diffusion step at which the best classification results are obtained for a given feature set. The corresponding training and testing time are recorded.

Table 1. Comparison of average classification accuracy (ACA), average training time (ATrTm) and average testing time (ATsTm) for the various feature sets based on wavelet transform and anisotropic diffusion using the optimal number of diffusion step (DS) for Brodatz dataset.

\begin{tabular}{|l|l|l|l|l|l|l|}
\hline $\begin{array}{l}\text { Sl. } \\
\text { No. }\end{array}$ & Feature set & DS & $\begin{array}{l}\text { ACA } \\
\text { (\%) }\end{array}$ & $\begin{array}{l}\text { ATrTm } \\
\text { (sec.) }\end{array}$ & $\begin{array}{l}\text { ATsTm } \\
\text { (sec.) }\end{array}$ & $\begin{array}{l}\text { No. of } \\
\text { features }\end{array}$ \\
\hline 1 & F1 & 9 & 79.30 & 3.71 & 0.24 & 15 \\
\hline 2 & F2 & 8 & 92.89 & 35.92 & 2.25 & 84 \\
\hline 3 & F3 & 7 & 84.69 & 3.06 & 0.19 & 15 \\
\hline 4 & F4 & 1 & 86.17 & 4.28 & 0.27 & 15 \\
\hline 5 & F5 & 1 & 73.44 & 3.39 & 0.22 & 12 \\
\hline 6 & F6 & 10 & 92.11 & 4.20 & 0.27 & 18 \\
\hline 7 & F7 & 9 & 42.19 & 4.01 & 0.25 & 12 \\
\hline 8 & F8 & 1 & 39.84 & 1.54 & 0.10 & 6 \\
\hline 9 & F9 & 1 & 19.84 & 1.37 & 0.09 & 15 \\
\hline 10 & F1+F3 & 9 & 93.20 & 4.61 & 0.29 & 30 \\
\hline 11 & F1+F3+F4 & 7 & 95.78 & 6.32 & 0.40 & 45 \\
\hline $\mathbf{1 2}$ & F1+F3+F4+F5 & $\mathbf{1 0}$ & $\mathbf{9 8 . 7 5}$ & $\mathbf{8 . 1 9}$ & $\mathbf{0 . 5 1}$ & $\mathbf{5 4}$ \\
\hline 13 & F1+F3+F4+F5+F6 & 9 & 97.58 & 8.20 & 0.52 & 72 \\
\hline 14 & F1+F3+F4+F5+F6+F7 & 9 & 96.41 & 8.61 & 0.54 & 84 \\
\hline 15 & F1+F3+F4+F5+F6+F7+F8 & 9 & 94.84 & 8.83 & 0.55 & 90 \\
\hline 16 & F1+F3+F4+F5+F6+F7+F8+F9 & 4 & 36.09 & 7.48 & 0.47 & 105 \\
\hline 17 & F1+F3+F5+F6 & 9 & 98.36 & 5.45 & 0.34 & 57 \\
\hline 18 & F3+F4+F5+F6 & 1 & 97.73 & 6.80 & 0.43 & 57 \\
\hline 19 & F1+F4+F5+F6 & 10 & 98.28 & 8.64 & 0.54 & 57 \\
\hline 20 & F6+F7+F8+f9 & 4 & 35.78 & 3.90 & 0.25 & 51 \\
\hline 21 & F1+F3+F5 & 10 & 98.36 & 6.33 & 0.40 & 39 \\
\hline 22 & F1+F3+F6 & 9 & 97.42 & 5.10 & 0.32 & 48 \\
\hline 23 & F1+F4+F5 & 6 & 97.81 & 6.91 & 0.44 & 39 \\
\hline 24 & F3+F4+F5 & 10 & 98.05 & 8.79 & 0.55 & 39 \\
\hline 25 & F6+F7 & 10 & 94.06 & 5.19 & 0.33 & 30 \\
\hline 26 & F4+F5 & 6 & 97.66 & 7.47 & 0.47 & 24 \\
\hline 27 & F3+F4 & 1 & 93.20 & 4.14 & 0.26 & 30 \\
\hline 28 & F5+F6 & 10 & 97.34 & 6.27 & 0.40 & 27 \\
\hline 29 & F8+F9 & 1 & 22.58 & 1.42 & 0.09 & 21 \\
\hline 30 & F2+F4 & 9 & 86.88 & 41.73 & 2.61 & 99 \\
\hline & & & & & & \\
\hline
\end{tabular}

It is observed from the Table 1 that the single feature set (F1 to F9) gives poor classification accuracy except F2 and F6. Even though the feature set F2 based on Haralick features gives $92.89 \%$ of classification accuracy, it is computationally expensive. The same observation is 
International Journal on Cybernetics \& Informatics (IJCI) Vol. 5, No. 1, February 2016

verified for the combination of feature sets F2+F4. Hence, F2 is not considered with other combinations of feature set. The combination of feature set gives improved classification accuracy compared to the feature set considered alone. Hence, the accuracy of classification depends on the type of features used.

It is also observed from the Table 1 that the feature sets F1, F3, F4, F5 and F6 are dominant feature sets. These feature sets give better classification accuracy in combinations. The optimal average classification accuracy of $98.75 \%$ is observed for $\mathrm{F} 1+\mathrm{F} 3+\mathrm{F} 4+\mathrm{F} 5$ feature set (Sl. No. 12) at the tenth diffusion step with average training time of $8.19 \mathrm{sec}$. and average testing time of 0.51 sec.

The proposed method is experimented on same dataset [33] as used in [8] and [19] so that, results can be compared. The methods NSCT and LDBP used in [8] and [19] are briefly described in Appendix. The Table 2 shows comparison of average classification accuracy attained by the proposed method and other methods in the literature on Brodatz dataset.

Table 2. Comparison of average classification accuracy (\%) obtained by the proposed method and other methods in the literature $[8,19]$ on Brodatz dataset.

\begin{tabular}{|l|l|l|l|}
\hline \multirow{2}{*}{ Image no. } & \multicolumn{3}{|l|}{ Average classification accuracy (\%) } \\
\cline { 2 - 4 } & $\begin{array}{l}\text { Method in [8] } \\
\text { (NSCT \& LDBP) }\end{array}$ & $\begin{array}{l}\text { Method in [19] } \\
\text { (PDE \& LDBP) }\end{array}$ & $\begin{array}{l}\text { Proposed method } \\
\text { (WT \& PDE) }\end{array}$ \\
\hline D104 & 100 & 100 & 100 \\
\hline D11 & 100 & 96.25 & 96.25 \\
\hline D16 & 100 & 100 & 100 \\
\hline D21 & 100 & 100 & 100 \\
\hline D24 & 100 & 98.75 & 98.75 \\
\hline D29 & 100 & 100 & 100 \\
\hline D3 & 100 & 95 & 100 \\
\hline D36 & 87.5 & 98.75 & 95 \\
\hline D4 & 100 & 100 & 97.5 \\
\hline D51 & 100 & 97.5 & 95 \\
\hline D52 & 100 & 100 & 97.5 \\
\hline D6 & 100 & 100 & 100 \\
\hline D68 & 100 & 100 & 100 \\
\hline D71 & 100 & 97.5 & 100 \\
\hline D75 & 100 & 100 & 100 \\
\hline D82 & 87.5 & 100 & 100 \\
\hline $\begin{array}{l}\text { Average classification } \\
\text { accuracy (\%) }\end{array}$ & 98.43 & 98.98 & 98.75 \\
\hline $\begin{array}{l}\text { Average training } \\
\text { time (sec.) }\end{array}$ & 278.50 & 14.92 & 8.18 \\
\hline $\begin{array}{l}\text { Average testing } \\
\text { time (sec.) }\end{array}$ & 12.42 & 0.93 & 0.51 \\
\hline
\end{tabular}

It is observed from the Table 2 that the average classification accuracy of the proposed method is improved as compared to the method based on NSCT and LDBP [8]. It is marginally less compared to the method based on PDE and LDBP [19]. The training time and testing time of the proposed method is considerably reduced. Thus, the proposed method is effective and computationally less expensive. 


\section{Conclusions}

In this paper, a novel method of PDE based statistical features for texture analysis using wavelet transform is proposed and is implemented on Brodatz dataset. The proposed method is compared with the other methods in the literature $[8,19]$. Following conclusions can be made from the experimentation:

- Better classification accuracy using simple statistical descriptors is achieved.

- Combination of feature sets improves the average classification accuracy.

- Feature set F2 (that computes the Haralick features) and other combination of feature sets containing F2 (F2+F4) is observed to be computationally expensive.

- Most feature sets are computationally inexpensive making it suitable for real time applications.

- The classification accuracy can be increased with more sophisticated classifier.

- The computational cost is reduced significantly as compared to other methods in the literature, while better classification accuracy is attained.

\section{ACKNOWLEDGEMENTS}

The authors are grateful to the reviewers for their valuable comments and suggestion, which substantially improved the quality of the paper.

\section{REFERENCES}

[1] Rosenfeld, A. (1976) Digital Picture Analysis, Springer Verlag, Berlin.

[2] Tuceryan, M. \& Jain, A.K. (1994) Texture Analysis handbook of Pattern Recognition and Computer Vision.

[3] Haralick, R.M., Shanmuga, K. \& Dinstein I., (1973) “Textural features for image classification”, IEEE Transactions on Systems, Man and Cybernetics, Vol. 3, pp 610-621.

[4] Tamura H., Mori, S., \& Yamawaki, T., (1978) "Textures Corresponding to Visual Perception”, IEEE Trans. syst. Man Cybern, Vol. 8, No. 6, pp 460-473.

[5] Hiremath, P.S., \& Shivashankar, S., (2008) "Wavelet based co-occurrence histogram features for texture classification with an application to script identification in a document image", Pattern Recognition Letters No. 29, pp 1182-1189.

[6] Smith, J. R., \& Chang, S. F., (1997) "Visually searching the web for content", IEEE Multimedia Magazine Vol. 4, No. 3, pp 12-20. [Columbia U. CU/CTR Technical Report 459-96-25].

[7] Smith, J. R., \& Chang, S. F., (1994) "Transform features for texture classification and discrimination in large image databases", in Proc. IEEE Int. Conf. on Image Proc.

[8] Hiremath, P. S., \& Bhusnurmath, Rohini A., (2013) “Texture Image Classification Using Nonsubsampled Contourlet Transform and Local Directional Binary Patterns”. Int. Journal of Advanced Research in Computer Science and Software Engineering, Vol. 3, No. 7, pp 819-827.

[9] Hiremath, P. S., \& Bhusnurmath, Rohini A., (2014) "A Novel Approach to Texture Classification using NSCT and LDBP”. IJCA Special Issue on Recent Advances in Information Technology, No. 3, pp 36-42 (ISBN-973-93-80880-08-3).

[10] Hiremath, P. S., \& Bhusnurmath, Rohini A., (2013) "Nonsubsampled Contourlet Transform and Local Directional Binary Patterns for Texture Image Classification Using Support Vector Machine". Int. Journal of Engineering Research and Technology, Vol. 2, No. 10, pp 3881-3890.

[11] Thyagarajan, K. S., Nguyen, T., \& Persons, C., (1994) "A maximum likelihood approach to texture classification using wavelet transform”, in Proc. IEEE Int. Conf. on Image Proc.

[12] Laws, K. I., (1979) “Texture energy measures," in Proc. Image Understanding Workshop, pp 47-51.

[13] Lendaris, G. O. \& Stanley, G. L., (1970) "Diffraction pattern sampling for automatic pattern recognition," in Proc. IEEE, Vol. 58, pp 198-216.

[14] Mandelbrot, B. B., (1982) The Fractal Geometry of Nature, San Francisco, CA, Freeman. 
International Journal on Cybernetics \& Informatics (IJCI) Vol. 5, No. 1, February 2016

[15] Perona, P., \& Malik, J., (1990) "Scale-Space and Edge Detection Using Anisotropic Diffusion", IEEE transaction on pattern analysis and machine intelligence. Vol.12, No.7, pp 629-639.

[16] Witkin, A. P., (1983) “Scale space filtering”, Proc. Int. Joint Conf. Artificial Intelligence pp 10191023.

[17] Hiremath, P. S., \& Bhusnurmath, Rohini A., (2014) “Texture Classification Using Anisotropic Diffusion and Local Directional Binary Pattern Co-Occurrence Matrix". Proceedings of the Second International conference on Emerging Research in Computing, Information, Communication and Applications (ERCICA 2014), No. 2, pp 763-769, Aug. 2014. ISBN: 9789351072621, Elsevier Publications 2014.

[18] Hiremath, P. S., \& Bhusnurmath, Rohini A., (2014) "RGB - Based Color Texture Image Classification Using Anisotropic Diffusion and LDBP”. Multi-disciplinary Trends in Artificial Intelligence, 8th International Workshop, MIWAI 2014, M.N. Murty et al. (Eds.)LNAI 8875, pp 101111, DOI 10.1007/978-3-319-13365-2_10, Springer International Publishing Switzerland 2014.

[19] Hiremath, P. S., \& Bhusnurmath, Rohini A., (2015) "Diffusion Approach For Texture Analysis Based On LDBP,” Int. Journal of Computer Engineering and Applications, Vol. 9, No. 7, Part I, pp 108-121.

[20] Randen, T., \& Husøy, J., (1999) "Filtering for Texture Classification: A Comparative Study", IEEE Trans. Pattern Analysis and Machine Intelligence, Vol. 21, No. 4, pp 291-310.

[21] Teuner, A., Pichler, O., \& Hosticks, B., (1995) "Unsupervised Texture Segmentation of Images Using Tuned Matched Gabor Filters”, IEEE Trans. Image Processing, Vol. 4, No. 6, pp 863-870.

[22] Daubechies, I., (1992) Ten Lectures on Wavelets, SIAM, Philadelphia, PA.

[23] Mallat, S. G., (1989) "A theory of multiresolution signal decomposition: The wavelet representation", IEEE transactions on pattern analysis and Machine intelligence, No. 11, pp 674-693.

[24] Ojala, T., \& Pietikäinen, M, (2004) Texture Classification, Machine Vision and Media Processing Unit, University of Oulu, Finland.

[25] Weszka, J. S., Dyer, C. R., Rosenfield, A., (1976) "A Comparative Study of Texture Measures for Terrain Classification", IEEE Transactions on Systems, Man. \& Cybernetics, Vol. 6. Pp 269-285,

[26] Srinivasan, G. N., \& Shobha, G., (2008) "Statistical Texture Analysis", Proc. Of World Academy Of Science, Engineering And Technology, Vol. 36, pp 1264-1269.

[27] Amadasun, M. \& King, R., (1989) "Texural Features Corresponding to Texural Properties", IEEE Transactions on Systems, Man, and Cybernetics, Vol. 19, No. 5, pp 1264-1274

[28] Chung-Ming, Wu., \& Yung-Chang, Chen, (1992) "Statistical Feature Matrix for Texture Analysis", CVGIP: Graphical Models and Image Processing, Vol. 54, No. 5, pp 407-419.

[29] Laws, K. I., (1980) "Rapid Texture Identification", SPIE, Vol. 238, pp 376-380.

[30] Haralick, R. M., \& Shapiro, L. G., (1992) Computer and Robot Vision Vol. 1, Addison-Wesley.

[31] Rosenfeld, A., \& Weszka, J., (1980) "Picture Recognition", in Digital Pattern Recognition, K. Fu (Ed.), Springer-Verlag, pp 135-166.

[32] Duda, R. O., Hart, P. E., \& Stork, (2001) Pattern Classification, Wiley publication, New York.

[33] Brodatz, P., (1966) Textures: A Photographic Album of Artists and Designers. Dover publication, New York.

[34] Weickert, J., (1998) Anisotropic Diffusion in Image Processing. ECMI Series, Teubner -Verlag.

[35] Coifman, R. R., \& Donoho, D. L., (1995) “Translation Invariant Denoising in Wavelets and Statistics", Lecture Notes in Statistics, pp 125-150.

[36] Cunha, A. L., Zhou, J., \& Do, M. N., (2006) “The Nonsubsampled Contourlet Transform: Theory, Design and Applications”, IEEE Trans. Image Processing, Vol. 15, No.10, pp 3089-3101.

\section{APPENDIX}

\section{Anisotropic diffusion}

The Partial differential equation (PDE) [19] techniques have been widely used for enhancing images. The diffusion process is equivalent to a smoothing process with a Gaussian kernel (linear diffusion). A major drawback of the linear diffusion is its uniform filtering of local signal features and noise. This problem was addressed by Perona and Malik [15], who proposed a nonlinear diffusion process, where diffusion can take place with a variable diffusion in order to control the smoothing effects [34] is represented by Eq. (1):

$$
p(\|\nabla I\|)=e^{-(\|\nabla I\| / K)^{2}}
$$


where $p(\nabla I)$ is the conduction function, $\nabla I$ is the gradient magnitude and the parameter $K$ controls the conduction.

It was demonstrated [15] that anisotropic diffusion clearly outperforms the canny edge detector, making image boundaries sharp. The Figure A1 represents the anisotropic diffusion on Brodatz texture image D104 of size 64 x 64. The row 1 represents the effect of anisotropic diffusion resulting in smoothing of image (cartoon approximation). The row 3 is the texture component of the corresponding images in the row 1, obtained by the difference between original image and it's cartoon approximation. Row 2 and 4 depict the contour representations of images in row 1 and row 3 , respectively.

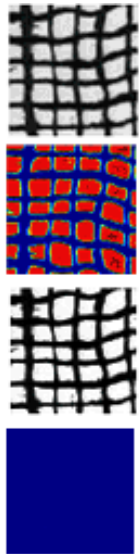

(a) I

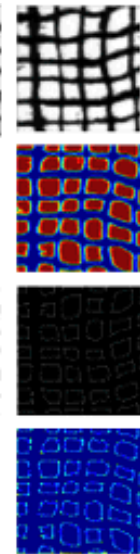

(b) $\mathrm{t}_{1}$

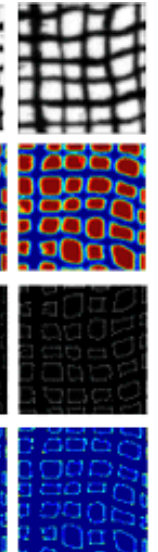

(c) $t_{2}$

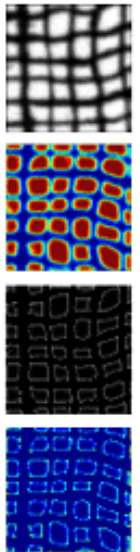

(d) $t_{3}$

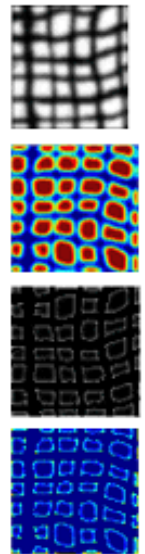

(e) $t_{4}$

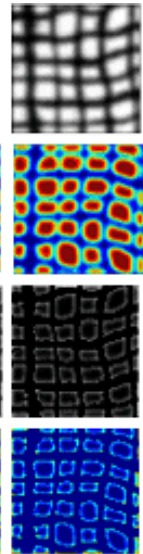

(f) $t_{5}$

Figure A1. Effect of anisotropic diffusion on Brodatz texture image D104 of size 64 x 64. Row 1 represents the smoothed images [(b) to (f)] of the original image I in (a) at successive diffusion steps $t_{1}$ through $t_{5}$. Row 3 represents the texture component of the image in row 1 . Row 2 and 4 represent the contour plot of the corresponding images in row 1 and row 3 , respectively [19].

\section{Local directional binary patterns}

The most important property of local directional binary patterns (LDBP) [8] is computational simplicity. The basic idea of LDBP is that, $3 \times 3$ kernel of image can be treated as basic texture region. The gray value of central pixel is compared with the gray values of eight pixels around it. The central gray pixel value is the threshold value. If the gray value of surrounding pixel is larger than gray value of central pixel, the surrounding pixel is marked as one otherwise zero. The binary values of all surrounding pixels can be obtained. All surrounding pixels are given different metrics. The metrics is multiplied with a binary value of surrounding pixels as shown in the Figure A2. Further, the sum of product of binary value and metrics of all surrounding pixels is set as the value of local directional binary pattern of central pixel. The value of local directional binary patterns of all pixels in an image can be obtained through such calculation neglecting the pixels of edges. The LDBP weight $f_{b}$ of the central pixel $\left(x_{c}, y_{c}\right)$ can be calculated using the Eq. (2) and Eq. (3).

$$
f_{b}\left(x_{c}, y_{c}\right)=\sum_{j=0}^{7} v\left(f_{j}-f_{c}\right) \cos (j * 45)
$$

where

$$
v(x)= \begin{cases}1 & x \geq 0, \\ 0 & x<0 .\end{cases}
$$


$f_{c}$ and $f_{j}$ are the pixel values at the center and $\mathrm{j}^{\text {th }}$ neighbor in $3 \times 3$ kernel of image. Finally, a matrix of LDBP weights is obtained.

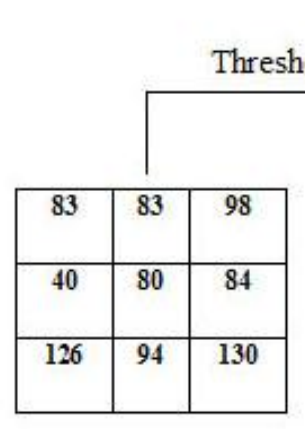

(a)

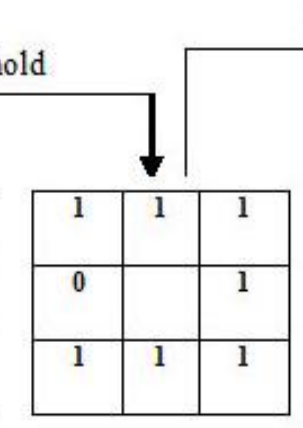

(b)
Multiply

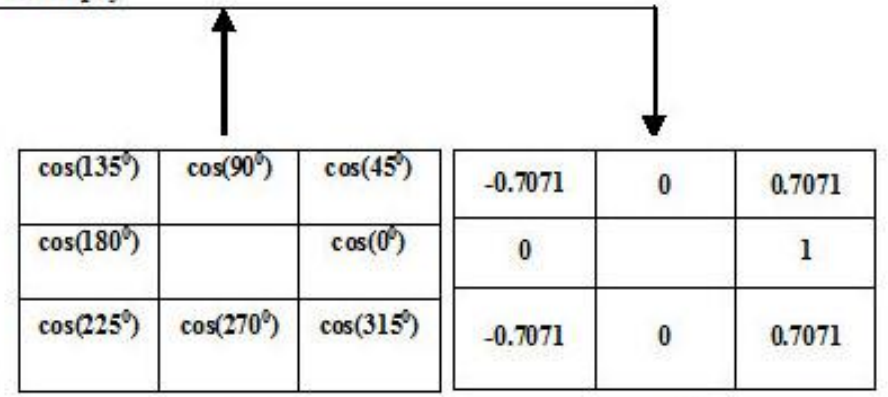

(c)

(d)

Figure A2. Transformation of neighborhood pixels to calculate central pixel weight in LDBP. (a) A sample neighborhood, (b) Resulting binary thresholded result, (c) LDBP mask, (d) Resultant weights after multiplying corresponding elements of (b) and (c) [18].

\section{Nonsubsampled contourlet transform}

An important feature of nonsubsampled contourlet transform (NSCT) is its stability with respect to shifts of the input signals [35]. The lack of shift invariance during image processing will cause pseudo Gibbs phenomena around singularities. In order to enhance directional selectivity and shift invariance and to get rid of the frequency aliasing of contourlet, Cunha et al. [36] presented a shift invariant version of the contourlet transform namely, NSCT. To obtain a shift invariant, directional multiresolution image representation, the NSCT is built upon iterated nonsubsampled filter banks. The NSCT combines nonsubsampled pyramids to provide multi scale decomposition and nonsubsampled directional filter bank (DFB) to provide directional decomposition. The two level NSCT decomposition is shown in the Figure A3.

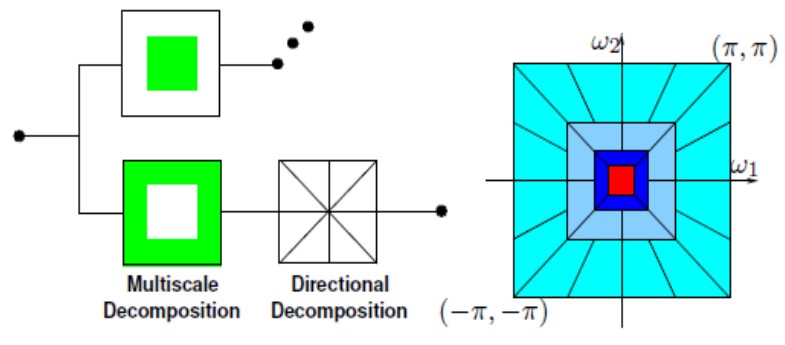

(a)

(b)

Figure A3. Nonsubsampled contourlet transform (a) Nonsubsampled filter bank (b) Idealized frequency partitioning [36].

The building block of the nonsubsampled pyramid is shown in the Figure A3(a). It is a two channel nonsubsampled filter bank which has no downsampling or upsampling and therefore is shift invariant. The ideal frequency response of a nonsubsampled DFB is shown in the Figure A3(b). The building block of a nonsubsampled DFB is a two channel nonsubsampled filter bank 
International Journal on Cybernetics \& Informatics (IJCI) Vol. 5, No. 1, February 2016

\section{AUTHORS}

Dr. P.S. Hiremath Professor, Department of Computer Science (MCA), KLE Technological University, BVBCET Campus, Hubli- 580031, Karnataka, India. His research areas of interest are Image Processing, Pattern Recognition, Computational Fluid Dynamics, Optimization Techniques, Data Mining, Web Mining and Knowledge Discovery Techniques. He has published 190 research papers in peer reviewed International Journals.

Rohini A. Bhusnurmath is currently pursuing her Ph.D. in Computer Science in the Department of P.G. Studies and Research in Computer Science at Gulbarga University, Gulbarga- 585106, Karnataka, India. Presently she is working as a Lecturer in the department of Computer Science, at Govt. P.U. College for Girls, Bijapur, Karnataka, India. Her research areas of interest are Image Processing and Pattern Recognition.
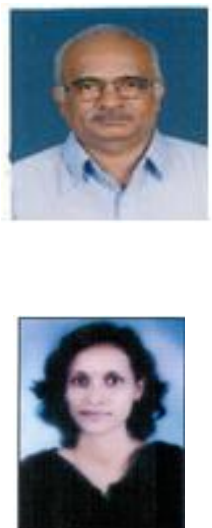